\title{
Revisión de los criterios audiométricos en el tratamiento de la hipoacusia neurosensorial mediante audífonos y prótesis auditivas implantables
}

Manuel Manrique, Adriano Valdivieso, David Ruba, Carlos Gimeno-Vilar, Lourdes Montes-Jovellar y Raquel Manrique Departamento de Otorrinolaringología. Clínica Universitaria de Navarra. Universidad de Navarra. Pamplona. Navarra. España.

La hipoacusia neurosensorial tiene alta incidencia en nuestra población. Sirva de ejemplo que el $50 \%$ de las personas mayores de 75 años tiene este tipo de discapacidad.

Los avances en los dispositivos utilizados para su tratamiento paliativo y su eficacia comprobada hacen necesaria la revisión de sus indicaciones y la descripción detallada de los sistemas audioprotésicos empleados. Éstos pueden ser clasificados en prótesis externas no implantables (audífonos) y prótesis implantables. El grupo de las prótesis implantables se subdivide a su vez en implantes activos de oído externo, implantes activos de oído medio, implantes cocleares e implantes auditivos de tronco cerebral (IATC). Las indicaciones establecidas para cada grupo audioprotésico se definen por la tipología y la topología de la enfermedad subyacente y por las características anatomofuncionales y socioculturales de cada paciente. En esta cuestión debe hacerse hincapié en el protagonismo del especialista a la hora de elegir y seguir el tratamiento. Como norma general, se procura favorecer el acceso del paciente hipoacúsico a su entorno sonoro realzando la comprensión de la palabra hablada restableciendo la binauralidad y, a la vez, se busca mantener la plasticidad de las vías auditivas centrales a través de la estimulación proporcionada por cualquiera de estos sistemas.

Se expone las indicaciones emergentes, ya sea en el campo de los implantes cocleares (estimulación bimodal, implantación en pacientes con audición residual, implantaciones bilaterales, etc.) o en el campo de los IATC, en pacientes con afección tumoral previamente tratada con radiocirugía $y$ en pacientes con trastornos no tumorales afectos de osificación coclear bilateral o malformaciones.

Palabras clave: Hipoacusia. Audífono. Implantes auditivos.

\section{Review of Audiometric Criteria in Treatment of Neurosensorial Deafness With Hearing Aids and Implantable Hearing Devices}

Sensorineural hearing loss has a high incidence in our population; as a matter of fact, $50 \%$ of people above 75 years of age suffer this impairment.

Due to the advances in the devices to alleviate this condition and their verified efficacy, it is now appropriate to review the indications for these devices and provide a detailed description of the audioprosthetic systems used. These systems can be classified as external non-implantable devices (hearing aids) and implantable prostheses. The latter can be sub-divided into active implants in the external ear or middle ear, cochlear implants, and auditory brainstem implants (ABI).

Indications for each group are determined by the type and location of the underlying condition as well as by the anatomic, functional, and social characteristics of each patient. It must be stressed that the selection and monitoring of the treatment is up to the specialist. Generally speaking, an attempt is made to facilitate the integration of the hypoacusic patients to their sound setting by enhancing their understanding of the spoken word and restoring binaurality, while at the same time, seeking to retain the plasticity of central auditory routes through the stimulation provided by any of these systems.

In the course of this review, we refer to newly-emerging indications in both the field of cochlear implants (bimodal stimulation, implantation in patients with residual hearing, bilateral implants, etc) and in the area of ABI in patients with tumoural disease previously treated with radiosurgery or patients with non-tumour pathologies presenting malformations or bilateral cochlear ossification.

Key words: Hearing loss. Hearing aids. Auditory implants.
Correspondencia: Dr. M. Manrique.

Avda. Pío XII, 36. Pamplona. España.

Correo electrónico: mmanrique@unav.es

Recibido el 9-10-2007.

Aceptado para su publicación el 10-10-2007.

\section{INTRODUCCIÓN}

Actualmente el tratamiento de la hipoacusia neurosensorial es de carácter paliativo y está basado en el empleo de 
audífonos y prótesis auditivas implantables. Son varias las razones por las cuales se ha estimado interesante hacer una revisión de las indicaciones de estos sistemas.

En primer lugar, por la elevada frecuencia de la hipoacusia neurosensorial en España. Baste exponer, a modo de ejemplo, la incidencia de la presbiacusia, la forma más frecuente de hipoacusia neurosensorial, que afecta a un $45 \%$ de las personas de 65 años de edad, porcentaje que se incrementa en edades más avanzadas y alcanza al 50\% de los mayores de 75 años ${ }^{1}$. Todo ello hace de esta afección una de las causas más frecuentes de consulta en la especialidad de otorrinolaringología.

En segundo lugar, porque cada vez son más satisfactorios los resultados obtenidos con el empleo de estos sistemas en el tratamiento de la hipoacusia neurosensorial. Si a ello unimos el crecimiento exponencial en el número y en las formas de los dispositivos utilizados, queda justificado llevar a cabo una revisión actualizada de sus indicaciones que ayude al otorrinolaringólogo a seleccionar la medida terapéutica más adecuada para cada paciente en particular.

\section{DESCRIPCIÓN Y CLASIFICACIÓN}

\section{Audífonos o audioprótesis no implantables}

Un audífono básicamente está constituido por un micrófono, un amplificador y un altavoz. Su objetivo es producir una amplificación acústica que permita obtener una curva audiométrica lo más próxima a la normalidad y proporcione una buena inteligibilidad en la percepción de la palabra hablada, evitando en la medida de lo posible cualquier tipo de distorsión.

De una forma sucinta se puede encontrar los siguientes tipos de audífonos ${ }^{2}$ :

- Audífonos retroauriculares: se ubican detrás de la oreja. Pueden ser empleados en todo tipo de hipoacusias.

- Audífonos intraauriculares: se introducen en el conducto auditivo externo y se prescriben en hipoacusias de grado leve y moderado.

- Audífonos de petaca o de bolsillo: el micrófono y el amplificador están situados en una especie de "caja", y se mantienen conectados por medio de un cable a un auricular que se localiza en la entrada del conducto auditivo externo. Cada vez se los emplea menos y cubren el mismo espectro de hipoacusias que los audífonos retroauriculares.

Si consideramos las características técnicas de los audífonos, destacan tres tipos: a) audífonos analógicos de control analógico; $b$ ) audífonos analógicos de control digital, y c) audífonos digitales.

\section{Prótesis auditivas implantables}

\section{Implantes de oído externo}

Estos implantes están compuestos por un sistema de pequeños tubos de titanio que comunican la región retroauricular con la luz del conducto auditivo externo a nivel de su porción cartilaginosa. Al extremo posterior de este tubo, se adapta un pequeño audífono que permanece casi oculto en la región retroauricular. Este sistema permite no ocluir el conducto auditivo externo, con lo que se evita los efectos de retroalimentación. Para su implantación precisa una cirugía sencilla con anestesia local, de carácter totalmente reversible ${ }^{3}$.

\section{Implantes activos de oído medio}

Los implantes activos de oído medio (IAOM) son dispositivos electrónicos que se implantan, total o parcialmente, mediante cirugía con el fin de corregir una pérdida auditiva con la excitación vibrátil de la cadena osicular ${ }^{3}$. De manera esquemática, un implante activo de oído medio consta de un micrófono, un audioprocesador, una batería, una unidad receptora (estos tres últimos componentes en los sistemas totalmente implantables reciben el nombre de módulo principal) y un transductor. En la tabla I se representan los principales tipos de IAOM $^{4-12}$.

Estos dispositivos presentan una serie de virtudes: evitan los problemas de retroalimentación, por elevada que sea la intensidad de estimulación, proporcionan una audición natural, con aceptables niveles de discriminación en ambiente ruidoso, anulan las molestias o contraindicaciones que plantean las enfermedades del conducto auditivo externo, ya que no lo ocupan, y para un buen número de pacientes, especialmente mujeres, proporcionan resultados estéticamente aceptables, sobre todo cuando se trata de sistemas totalmente implantables. Por otro lado los IAOM también tienen inconvenientes: precisan para su colocación de un procedimiento quirúrgico que la mayor parte de las veces requiere anestesia general, su implantación plantea potenciales riesgos de daño para la cadena osicular, ya que, en caso de fallo técnico de los componentes implantados se hace necesaria una reintervención quirúrgica para la sustitución del implante, y algunos dispositivos limitan la práctica de resonancia magnética y la aplicación de terapia electroconvulsiva y radioterapia sobre la región craneal.

\section{Implantes cocleares}

Su funcionamiento se basa en la transformación de sonidos y ruidos ambientales en energía eléctrica, capaz de ac-

TABLA I. Principales tipos de IAOM

\begin{tabular}{|c|c|c|c|}
\hline Transductor & Investigadores & Compañía & Implantación \\
\hline Piezoeléctrico & Yanagihara et al ${ }^{4}$ & Rion & Parcial \\
\hline Piezoeléctrico & Welling et al ${ }^{5}$ & St. Croix Medical & Total \\
\hline Piezoeléctrico & Zenner et al ${ }^{6}$ & IMPLEX & Total \\
\hline Electromagnético & Kartush et $\mathrm{al}^{7}$ & $\begin{array}{l}\text { Smith Nephew } \\
\text { Richards }\end{array}$ & Parcial \\
\hline Electromagnético & Perkins $^{8}$ & Resound & Parcial \\
\hline Electromagnético & Maniglia et $a l^{9}$ & Wilson Greatbach & Parcial \\
\hline Electromagnético & Baker et al ${ }^{10}$ & & Parcial \\
\hline Electromecánico & Gan et $\mathrm{al}^{11}$ & $\begin{array}{l}\text { Vibrant Sound } \\
\text { Bridge }\end{array}$ & Parcial \\
\hline Electromecánico & Frederickson et $\mathrm{al}^{12}$ & 2 Otologics & Parcial-total \\
\hline
\end{tabular}


tuar sobre las aferencias del nervio coclear, lo que determina una sensación auditiva ${ }^{3,13}$.

En esencia, un implante coclear consta de un micrófono, que se ubica en una carcasa similar a la de un audífono que permanece colgada de la oreja en la región retroauricular o a veces se sitúa en el mismo transmisor. Las señales recogidas son transmitidas a un procesador, el cual puede estar incorporado a la misma carcasa del micrófono (procesador retroauricular) o puede ser un elemento aparte conectado por un cable (procesador corporal). Junto a este procesador se sitúa un compartimento que alberga las baterías que proporcionan energía al sistema. El procesador tiene la misión de codificar las señales y enviarlas a un transmisor o bobina, localizado en la superficie de la piel en la región temporoparietal, que se mantiene en dicha posición por la atracción magnética generada entre dos imanes, uno ubicado en el mismo transmisor y otro en el receptor-estimulador. El transmisor emite señales por radiofrecuencia modulada que atraviesan la piel, y éstas son recogidas por una antena y un receptor-estimulador ya colocados, mediante cirugía, en la superficie del hueso craneal, bajo la piel de la región retroauricular. Este último elemento descodifica el mensaje y lo envía a cada uno de los electrodos emplazados, generalmente, dentro de la cóclea para así estimular el nervio coclear.

Existen diferentes tipos de implantes cocleares, que se puede clasificar en función a tres criterios: $a$ ) ubicación de los electrodos (intracocleares o extracocleares); $b$ ) número de canales de estimulación (uno o múltiples), y c) forma de tratar la señal sonora (estrategias de codificación basadas en extraer o no los formantes del sonido y la voz humana).

\section{Implantes auditivos de tronco cerebral}

El concepto de esta prótesis es similar al del implante coclear, salvo que el diseño de los electrodos y la técnica quirúrgica para su colocación tienen como objetivo los núcleos cocleares, en vez de la escala timpánica de la cóclea ${ }^{3}$.

Esta prótesis consta de un grupo de electrodos, un receptor-estimulador, una antena (todos ellos conforman los elementos internos o implantados), un transmisor, un procesador de la palabra y un micrófono (elementos externos, no implantados). Si bien los componentes de esta prótesis son parecidos a los de un implante coclear, la principal diferencia con respecto a éste se encuentra en los electrodos. $\mathrm{Su}$ forma se adapta a la anatomía de los núcleos cocleares y los electrodos se disponen en una cara de un portaelectrodos de forma rectangular $\mathrm{u}$ ovalado.

\section{INDICACIONES}

\section{Audífonos o prótesis no implantables}

No existe una normativa establecida para determinar cuál es el nivel de pérdida de audición a partir del cual resulta imprescindible la adaptación de una de estas prótesis (considerando los datos recogidos en la audiometría tonal liminar). En el caso de los niños se estima que existe una indicación absoluta para su prescripción cuando el umbral de audición medio es $\leq 40 \mathrm{~dB}$ HL en el oído mejor, promediando umbrales de frecuencias comprendidas entre 500 y $2.000 \mathrm{~Hz}$ en la audiometría tonal liminar. Sin embargo, en los adultos, con un desarrollo comunicativo y cognitivo ya adquirido, la indicación de audífonos es recomendable en hipoacusias de intensidad superior a moderada (41-70 dB $\mathrm{HL})$, necesaria en las de tipo severo (71-90 dB HL) e imprescindible en las de carácter profundo $(>90 \mathrm{~dB} \mathrm{HL})^{14}$. En este último caso se debe considerar claramente la opción de indicar un implante coclear (fig. 1).

El restablecimiento de la audición y, en este caso, la adaptación de los audífonos deben efectuarse lo más tempranamente posible. Esto es especialmente importante en los primeros años de vida, ya que el desarrollo de sus áreas corticales auditivas y la normal evolución de su lenguaje dependen de que reciban un estímulo auditivo adecuado ${ }^{15,16}$. Por ello la adaptación de audífonos, y en general cualquier tipo de prótesis auditiva, debe y puede efectuarse tan pronto como se conozca que tienen una deficiencia auditiva que reúne los criterios audiométricos señalados anteriormente para su indicación. Lafon señala que se puede originar graves problemas educativos si las hipoacusias profundas, severas y medias no son tratadas antes de los 24, 24 y 36 meses de vida, respectivamente ${ }^{17}$.

Como regla general, se debe procurar restablecer la biauralidad de la audición. Algunas de las ventajas que se puede obtener con la adaptación biaural respecto a la monoaural son: mejor localización de los sonidos, ausencia del efecto sombra de la cabeza, mejor discriminación del lenguaje, especialmente en ambientes ruidosos, y un efecto de sumación de aproximadamente $3 \mathrm{~dB}$.

Estimular bilateralmente, en especial en la infancia permite conservar las vías y centros auditivos de ambos hemisferios cerebrales.

La adaptación será biaural en la medida en que el rango dinámico y el grado de molestias de cada oído sean semejantes, los niveles de discriminación verbal sean prácticamente iguales y los umbrales de tonos puros por vía ósea no difieran más de $30 \mathrm{~dB}$ HL. En los casos en los que no se cumplan las mencionadas condiciones, se planificará una adaptación monoaural ${ }^{18}$.

La ganancia que aporta la prótesis debe conseguir que la curva audiométrica se asemeje lo más posible a la normal, a fin de obtener con ello una buena inteligibilidad de la palabra hablada. No obstante, es preciso considerar que cuando el porcentaje de discriminación inicial es inferior al $60 \%$ se estima que la adaptación protésica será difícil y los resultados, limitados ${ }^{17}$ (fig. 1).

Hay una serie de contraindicaciones para la prescripción de un audífono de vía aérea, que pueden clasificarse de la siguiente forma ${ }^{17}$ :

1. Relacionadas con el conducto auditivo externo (CAE):

- Absolutas: agenesia de CAE.

- Relativas: CAE estrecho de origen congénito o en presencia de exostosis, problemas cicatrizales o protrusiones del cóndilo de la mandíbula.

- Temporales: CAE obliterado (tapón de cera, tapón epidérmico) o intolerante por dermatitis crónicas.

2. Relacionadas con la membrana timpánica y los espacios de oído medio: 
- Relativas: cavidad abierta de mastoidectomía.

- Temporales: perforación no marginal activa desde un punto de vista infectivo-inflamatorio y perforación marginal sin o con formación de colesteatoma.
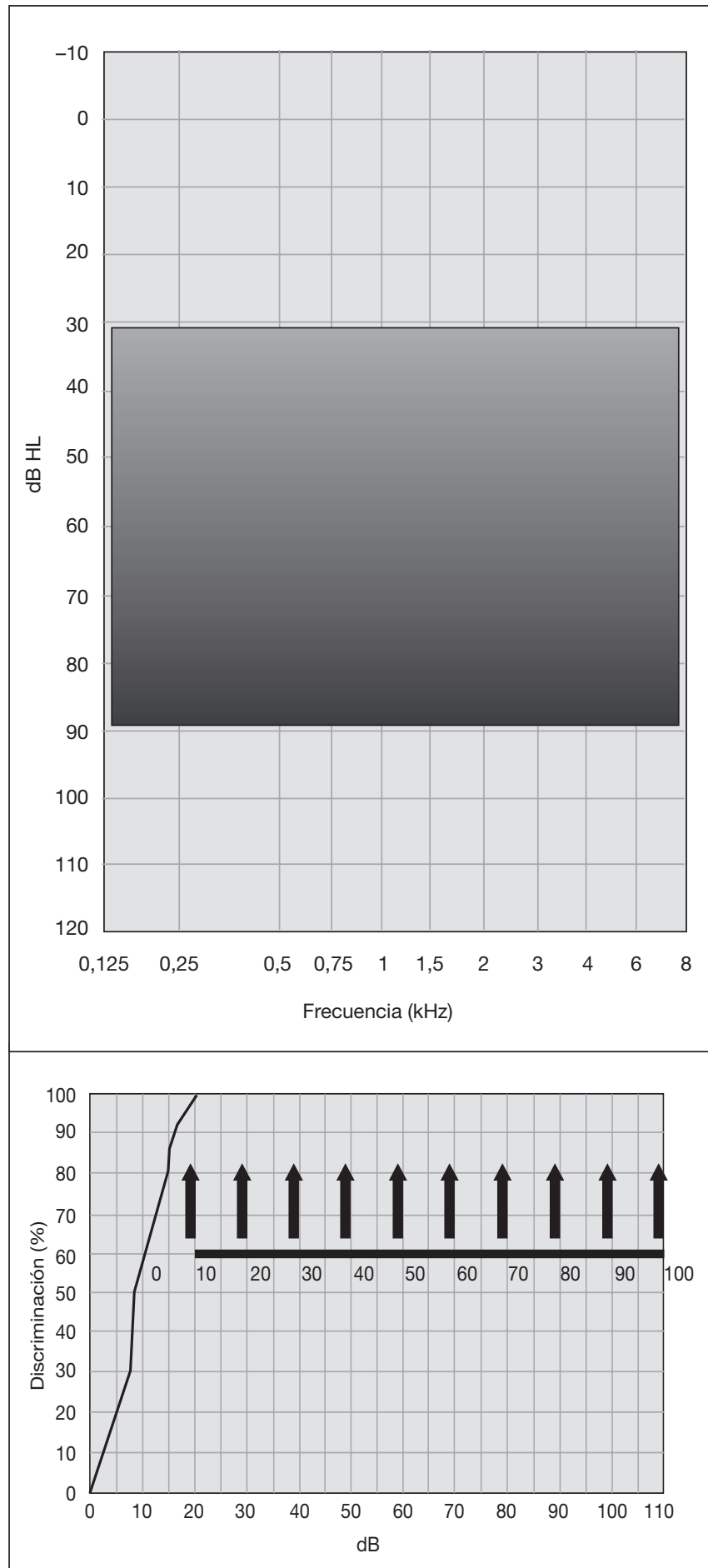

Figura 1. Criterios audiométricos para la indicación de audífonos. Sobre el audiograma se sobreimpone un área donde deben quedar incluidos los umbrales de conducción aérea determinados con audiometría tonal liminar. Asimismo, en el gráfico de logoaudiometría se marca el porcentaje de discriminación a partir del cual el pronóstico de la adaptación audioprotésica es favorable.
Los audífonos deben ser prescritos por los especialistas en otorrinolaringología, quienes además deberán controlar la evolución de los pacientes a los que se haya adaptado este tipo de audioprótesis. La adaptación de los audífonos se realiza profesionales especializados (audioprotesistas), que han de seleccionar la prótesis, llevar a cabo los oportunos reglajes y establecer las revisiones que sean necesarias para determinar su funcionamiento ${ }^{17}$. Es recomendable que el centro de adaptación de audífonos reúna una serie de condiciones, establecidas por un comité de expertos, que aseguren la calidad de la adaptación.

\section{Implantes de oído externo}

El dispositivo de oído externo tiene una ganancia máxima para las frecuencias situadas entre 2.000 y $6.000 \mathrm{~Hz}$, de aquí que sea un dispositivo diseñado especialmente para hipoacusias con pérdidas situadas sobre todo en las frecuencias agudas, con buena conservación de la audición en la gama de frecuencias graves y medias (fig. 2).

Las contraindicaciones fundamentales son: hipoacusia fluctuante, conductos auditivos muy estrechos, enfermeda-

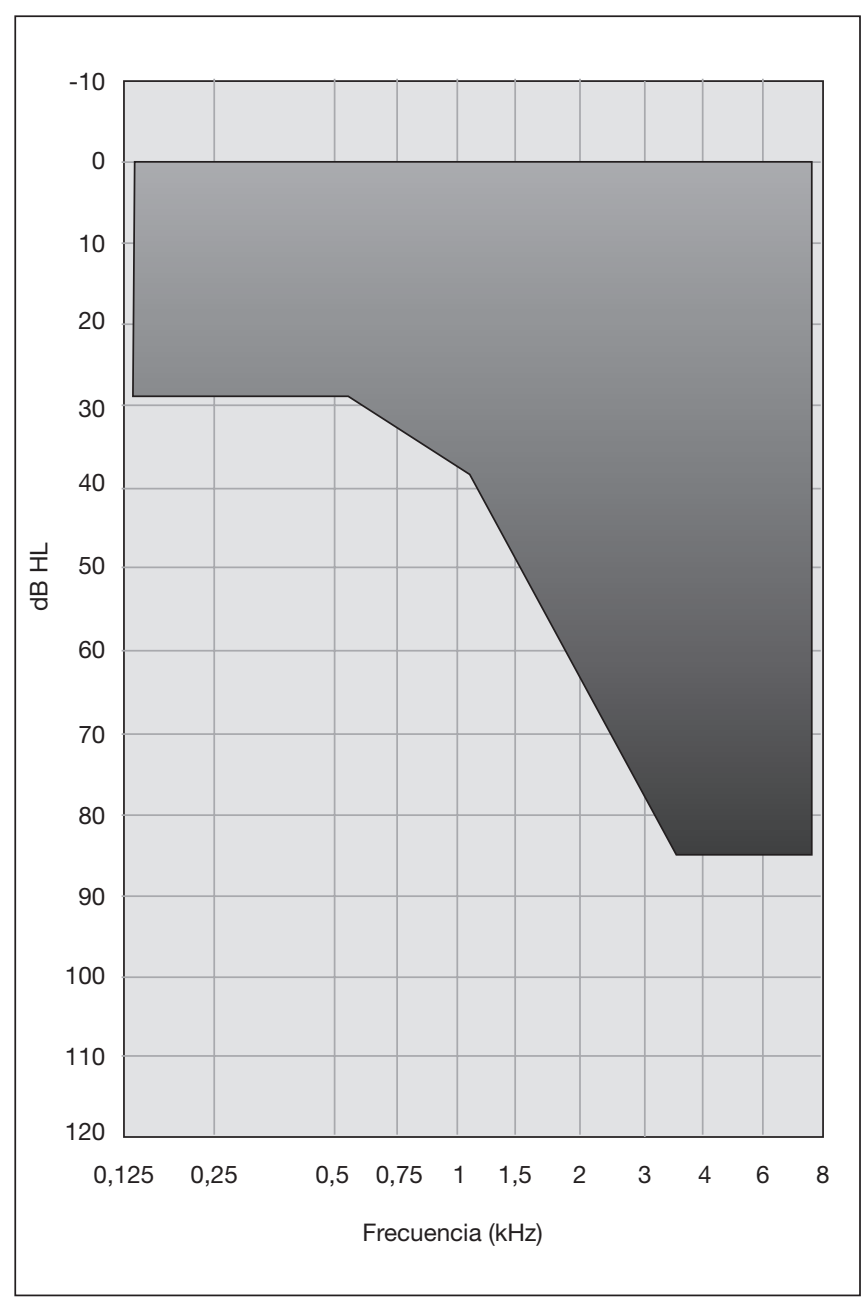

Figura 2. Criterios audiométricos para la indicación de implantes de oído externo. Sobre el audiograma se sobreimpone un área donde deben quedar incluidos los umbrales de conducción aérea determinados con audiometría tonal liminar. 
des cutáneas de la zona, otorreas crónicas, necesidad de emplear protectores acústicos y trabajos en ambientes muy contaminados.

\section{Implantes activos de oído medio}

La indicación básica que recordar son las hipoacusias moderadas-severas de tipo neurosensorial. Siguiendo las recomendaciones del "International consensus on middle ear implants" ${ }^{\prime 19}$, celebrado en Valencia en $2004^{19}$, se considera inapropiado indicarlos si el paciente no se beneficia de una prótesis auditiva de estimulación acústica aérea.

Los requisitos fundamentales para indicar estos dispositivos son: tener más de 18 años de edad; hipoacusia neurosensorial bilateral, con umbrales de conducción aérea en el rango de pérdida de moderada a severa; el umbral diferencial aéreo-óseo en las frecuencias de 500, 1.000, 2.000 y $4.000 \mathrm{~Hz}$ no debe de ser superior a $10 \mathrm{~dB}$, en 2 o más de esas frecuencias; discriminación del lenguaje superior al $50 \%$ empleando listas de palabras bisilábicas; funcionamiento y anatomía del oído medio normales; ausencia de cirugías previas en el oído medio; que no haya evidencia de altera- ciones retrococleares en la vía auditiva; insatisfacción comprobada en el uso de audífonos al menos durante 6 meses en el oído que se va a implantar; dado que estos implantes no producen oclusión del conducto auditivo externo, su indicación debe ser especialmente considerada en los casos que cursan con afección crónica del oído externo, como en las otitis externas crónicas, y expectativas realistas y ausencia de alteraciones vestibulares ${ }^{19-21}$.

Como se ha reseñado, estos implantes activos de oído medio están indicados en hipoacusias neurosensoriales de grado moderado a severo. No obstante, los criterios audiométricos pueden presentar determinadas variaciones para cada dispositivo en particular. En la figura 3 se recogen los criterios audiométricos para dos de los dispositivos comercializados en Europa con la marca CE.

\section{Implantes cocleares}

La mayoría de los autores coinciden en afirmar que los implantes cocleares están indicados en pacientes con hipoacusia neurosensorial profunda bilateral, que obtienen escasos beneficios en el uso de audífonos. Esta condición
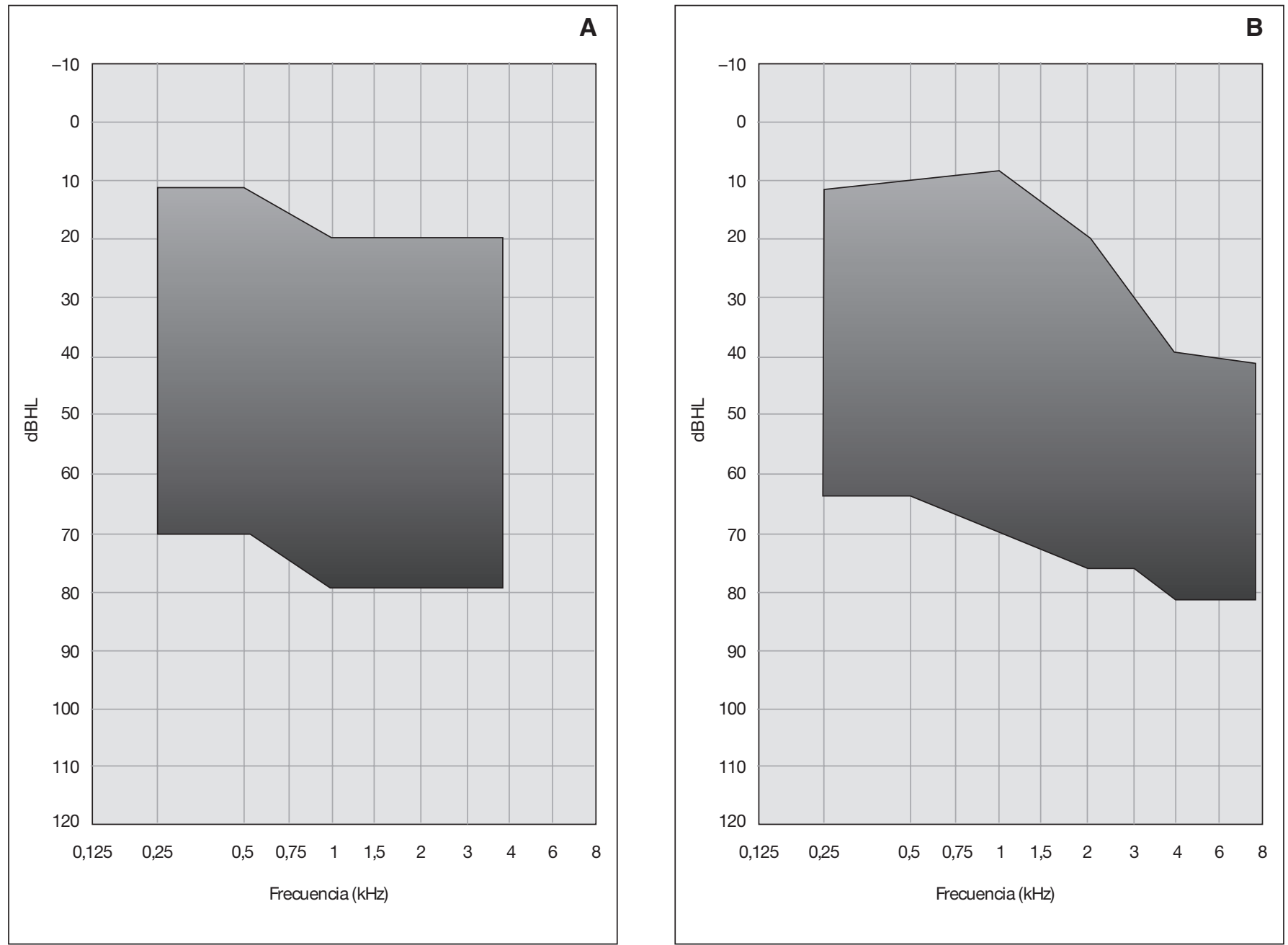

Figura 3. Criterios audiométricos para la indicación de implantes activos de oído externo. Sobre cada uno de los audiogramas se sobreimponen unas áreas donde deben quedar incluidos los umbrales de conducción aérea determinados con audiometría tonal liminar para los dispositivos MET de Otologics (A) y Vibrant Sound Bridge (B). 
corresponde a una amplia lista de causas congénitas o adquiridas que pueden ocurrir en las fases prelocutoria, perilocutoria o poslocutoria del desarrollo del lenguaje.

La evolución clínica posterior a la implantación depende de múltiples factores. Entre ellos, la etapa de aparición de la hipoacusia, la edad de implantación, las condiciones anatómicas de la cóclea y el propio desarrollo tecnológico de los implantes cocleares. Este último ha ido modificando con el tiempo los criterios de indicación de un implante coclear.

Los criterios audiométricos siguen siendo el parámetro más importante a la hora de seleccionar un candidato para implantes cocleares. Se expone a continuación los criterios establecidos y asimismo se refleja los emergentes que pueden servir de referencia en situaciones clínicas diferenciadas.

\section{Criterios audiométricos establecidos}

Considerando la población de candidatos poslocutorios adultos, según la Food and Drug Administration estadounidense, un implante coclear está indicado en las hipoacusias neurosensoriales bilaterales con umbrales auditivos $>70 \mathrm{~dB}$ para la media de las frecuencias 500, 1.000, $2.000 \mathrm{y}$ $4.000 \mathrm{~Hz}$, con audiometría tonal en campo libre con audífono superior a $55 \mathrm{~dB}$ en las mismas frecuencias y con una discriminación menor del $40 \%$ empleando listas abiertas de palabras con adecuado equipamiento audioprotésico a una intensidad de estimulación de $65 \mathrm{~dB} \mathrm{HL}^{22}$.

En el caso de los niños, según el mismo organismo, los criterios audiométricos son más restrictivos que en los adultos. Los implantes cocleares están indicados en hipoacusias neurosensoriales bilaterales con pérdidas medias $>90 \mathrm{~dB}$ HL $(500,1.000,2.000,4.000 \mathrm{~Hz})^{22}$. La FDA recomienda realizar la implantación después de los 12 meses de edad. Sus motivos son la dificultad de realizar pruebas conductuales de la audición, la inexistencia de pruebas objetivas para valorar los umbrales auditivos para todo el rango de frecuencias, el escaso tiempo de prueba con audífono y las enfermedades de oído medio concomitantes, que pueden dificultar el diagnóstico del grado de hipoacusia antes de los 18 meses. Sin embargo, a medida que ha aumentado la experiencia en el uso de implante coclear y han mejorado las técnicas de diagnóstico, la edad tiene una tendencia clara a disminuir. Ésta es factor determinante de los resultados tras la implantación debido a la existencia de un período crítico para la adquisición del lenguaje, el cual abarca los primeros años de vida. Por ello, actualmente el límite inferior de edad lo marca la seguridad diagnóstica para determinar el grado de hipoacusia ${ }^{23}$.

Existe un amplio número de "candidatos tradicionales" que cumplen estos criterios audiométricos. Sin embargo, en algunos de ellos es preciso considerar con especial detalle la indicación final de implantación ya que concurren factores de gran importancia pronóstica. Tal es el caso de:

- Adolescentes y adultos con hipoacusias de aparición prelocutoria ${ }^{24}$.

- Pacientes con malformaciones congénitas del oído interno o con diferentes grados de osificación coclear ${ }^{25,26}$.
- Candidatos con otras discapacidades relacionadas con la hipoacusia ${ }^{27}$.

\section{Criterios audiométricos emergentes}

Estimulación bimodal. Después de una serie de ensayos clínicos, hay una tendencia a realizar implantaciones en pacientes con hipoacusia neurosensorial severa en un oído e hipoacusia profunda en el otro. La experiencia clínica ha mostrado que estos pacientes utilizan de manera simultánea un implante coclear en el oído con menos audición y un audífono en el otro oído. A este paradigma de estimulación se lo denomina estrategia bimodal. Con ella, se ha comprobado que estos pacientes alcanzan estereofonía y mejor discriminación del lenguaje, tanto en ambiente de silencio como en ruido, en comparación con los obtenidos empleando audífonos o solamente un implante coclear ${ }^{28,29}$. El criterio audiométrico empleado sería el siguiente: hipoacusias neurosensoriales profundas en un oído y severas (71-90 dB) en el otro. La colocación del implante coclear se haría en el oído auditivamente peor ${ }^{30}$ (fig. 4).

Implantación en pacientes con audición residual. Existen pacientes con buena audición residual en frecuencias graves y caídas abruptas de la audición en frecuencias medias y agudas. Este grupo de pacientes suele tener una pobre discriminación de la palabra hablada que no mejora de forma sustancial con el uso de audífonos. En ellos, con el empleo de guías de electrodos especiales y una técnica quirúrgica depurada, que ha venido a denominarse atraumática, es posible preservar los restos de audición en el oído implantado. Esta circunstancia permite llevar a cabo, en el mismo oído y de forma simultánea, una estimulación eléctrica con el implante coclear y acústica con el audífono (estimulación híbrida). Los resultados obtenidos con este paradigma de estimulación indican que los pacientes son capaces de oír significativamente mejor, especialmente en ambiente de ruido, y alcanzar una percepción musical satisfactoria ${ }^{31}$. Si bien esta fórmula de tratamiento se encuentra aún en fase experimental y no existen criterios unificados, podríamos incluir a los pacientes para esta técnica cuando reúnan los criterios siguientes: a) 18 años de edad o mayor; $b$ ) pérdida de audición neurosensorial poslocutoria de severa a profunda en las frecuencias $>1.500 \mathrm{~Hz}$ y pérdida de audición neurosensorial poslocutoria de leve a moderada en las frecuencias $>500 \mathrm{~Hz}$, sin restricciones audiométricas para el oído contralateral (fig. 5); c) duración de la hipoacusia $<30$ años, y d) reconocimiento de palabras bisilábicas con ayuda (prótesis ajustada correctamente) en el oído que se va a implantar entre un 10 y un $50 \%$, en silencio y a $65 \mathrm{~dB}$ SSPL.

Implantes cocleares bilaterales. Diversos estudios muestran claros beneficios en pacientes sometidos a una implantación bilateral, pues los capacita para localizar sonidos, obtener un efecto de sumación, evitar el efecto sombra de la cabeza y mejorar la discriminación de la palabra en ambientes ruidosos ${ }^{28,32}$. El criterio audiométrico más extendido para la indicación simultánea o secuencial de implante coclear con carácter bilateral es una hipoacusia neurosen- 

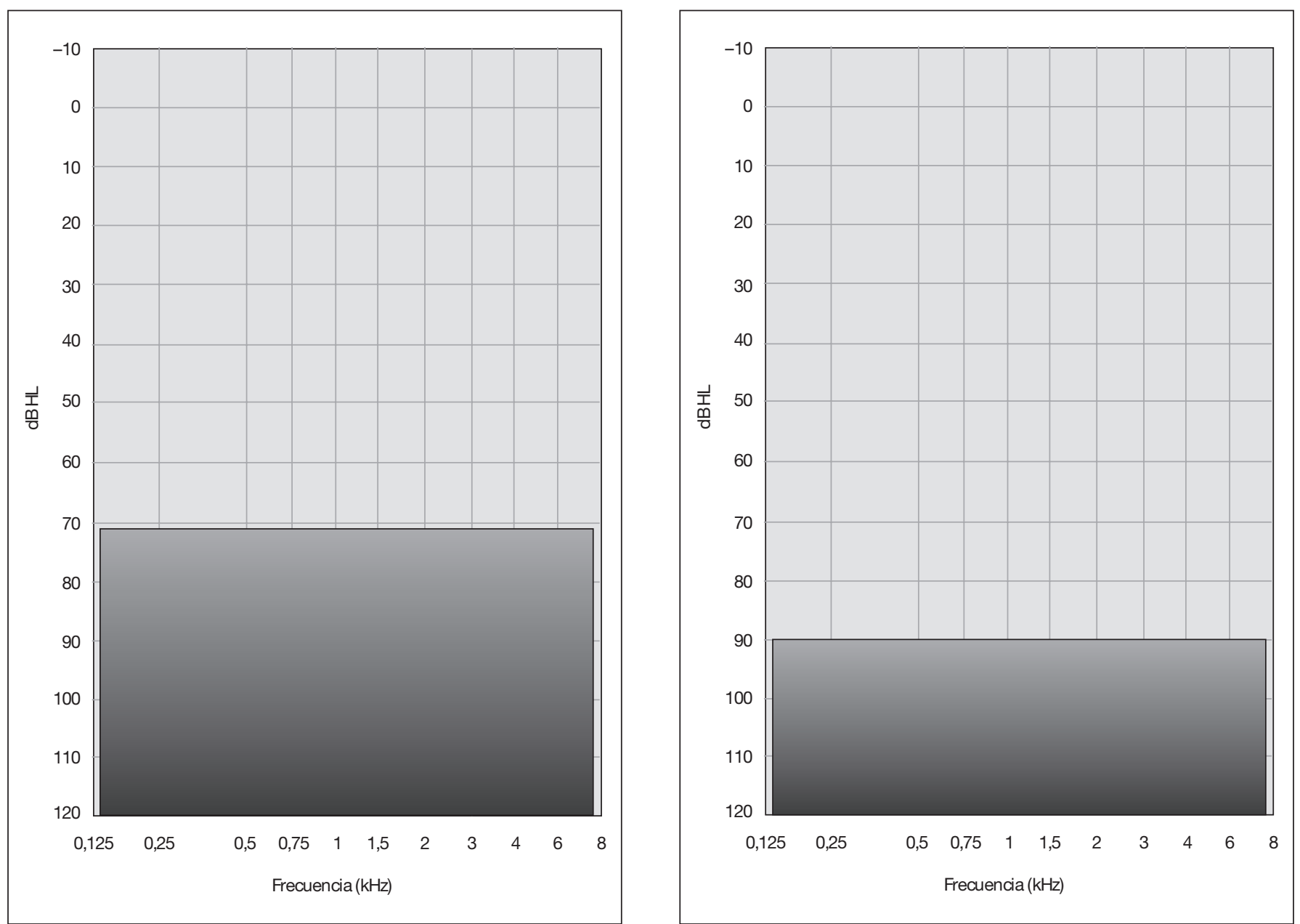

Figura 4. Criterios audiométricos para la indicación de un implante coclear en casos de estimulación bimodal. Se representan los audiogramas de los dos oídos. Sobre cada uno de ellos se sobreimponen las áreas donde deben quedar incluidos los umbrales de conducción aérea determinados con audiometría tonal liminar para el implante coclear (umbrales > $90 \mathrm{~dB} \mathrm{HL}$ ) y el audífono (umbrales 70-90 dB HL).

sorial profunda en los dos oídos, con escasa discriminación de palabras bisilábicas $(<40 \%)$ con el uso de audífonos adecuadamente adaptados.

\section{Implantes auditivos de tronco cerebral}

Los criterios de selección de candidatos para un implante auditivo de tronco cerebral (IATC) han ido variando con el tiempo. Durante años su indicación se ha centrado principalmente en el tratamiento de pacientes con formaciones tumorales a nivel de los dos nervios acústicos en el contexto de una neurofibromatosis tipo II. En esos casos, los criterios aceptados para la indicación de IATC se concretan en los siguientes puntos: $a$ ) pacientes diagnosticados de neurofibromatosis de tipo II que diera lugar a una severa disfunción bilateral del nervio acústico; $b$ ) 15 o más años de edad; c) la implantación podría tener lugar durante la primera extirpación de un tumor o en la segunda, correspondiente al otro lado, o siguiendo la extirpación bilateral de los tumores, o después del diagnóstico de disfunción bilateral del nervio acústico de origen traumático; con menos frecuencia, la implantación podría llevarse a cabo en un segundo tiempo quirúrgico, después de la extirpación de los neurofibro- mas en un primer tiempo; d) los candidatos deben reunir unas condiciones médicas y psicológicas que permitan la realización de cirugía con anestesia general y la adecuada colaboración en el período de rehabilitación auditiva después de la implantación; e) los pacientes han de ser preparados y motivados a participar en las regulares sesiones de programación, valoración y seguimiento, y f) no se especifica los criterios audiológicos debido a que la propia evolución natural de los tumores del ángulo pontocerebeloso, la usual vía de abordaje translaberíntica, empleada en su extirpación, y el origen traumático de la lesión bilateral del VIII par causan por sí solos una pérdida de la audición útil al paciente. No obstante, en los pacientes con audición útil, considerando los criterios de Briggs-Brackmann ${ }^{33}$ y de Doyle-Nelson ${ }^{34}$, se puede actuar selectivamente en nuestras indicaciones siguiendo este esquema:

- Tumores bilaterales $<1,5 \mathrm{~cm}$ con normoaudición bilateral: cirugía de resección unilateral conservadora por fosa media. Se realizará la implantación de un IATC en un segundo tiempo en función del resultado alcanzado en la primera cirugía y con respecto al control tumoral. 


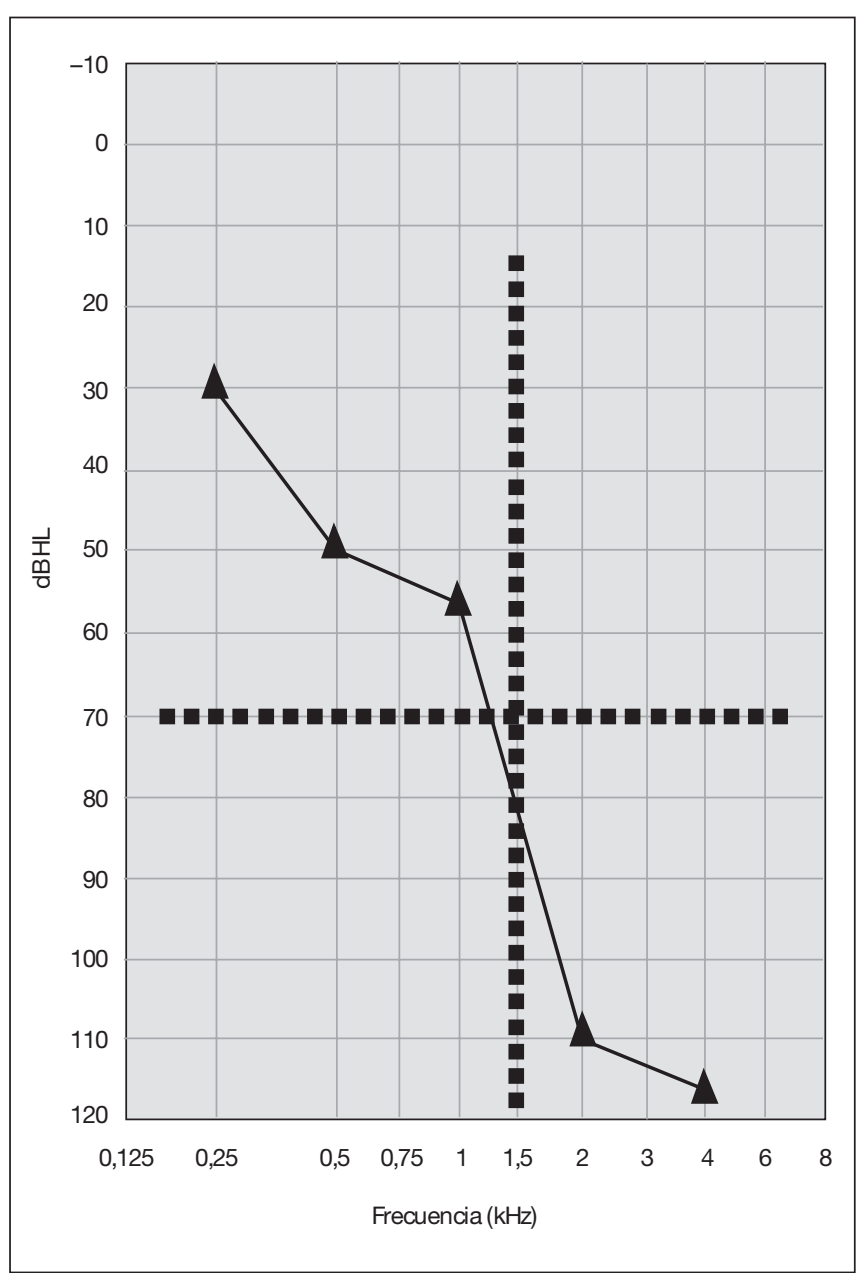

Figura 5. Criterios audiométricos para la indicación de un implante coclear en casos de audición residual. Se representa en el audiograma del oído que se va a implantar la vía aérea de una pérdida de audición de severa a profunda en las frecuencias superiores a $1.500 \mathrm{~Hz}$ y leve a moderada en las frecuencias $>500 \mathrm{~Hz}$.

- Tumor $<1,5 \mathrm{~cm}$ asociado a tumor $>2-3 \mathrm{~cm}$ contralateral, con audición útil: cirugía de resección del tumor mayor por vía translaberíntica más IATC y observación del segundo tumor y decisión terapéutica en función de la evolución.

- Tumores $>2-3 \mathrm{~cm}$ bilaterales: en ellos es raro no encontrar alteraciones auditivas. En estos casos se realiza una resección por vía translaberíntica más la colocación de un IATC.

\section{Indicaciones emergentes}

Tumores previamente tratados con radiocirugía. Inicialmente, el grupo de pacientes ya tratados con radiocirugía era excluido de la indicación de un IATC debido al potencial deterioro que la radiación producía en los núcleos cocleares $^{35-37}$. Sin embargo, este criterio está siendo revisado ante la experiencia de otros autores que han obtenido resultados auditivos después de la implantación de estos pacientes comparables con los registrados en otros no tratados previamente con radioterapia ${ }^{38,39}$.
Osificación bilateral coclear total. Por lo general, son muy escasos los resultados obtenidos en pacientes sometidos a implantaciones cocleares, en situaciones de una completa osificación coclear vinculada a una laberintitis meningíti$\mathrm{ca}^{40}$. Ello se debe a las dificultades que plantean la normal inserción de los electrodos del implante coclear y, especialmente, la relevante reducción de neuronas estimulables a nivel del ganglio espiral. Algunos autores han mostrado una mejoría relevante en la percepción de la palabra en un contexto abierto, en pacientes tratados con un IATC en el mismo oído que previamente había recibido un implante coclear $^{41,42}$. Estos datos, además de abrir una nueva indicación para los IATC en oídos con laberintitis osificantes muy avanzadas, arrojan unos satisfactorios resultados clínicos en la estimulación de una vía auditiva no alterada, como es el caso de la neurofibromatosis tipo II. Por ello, se abren nuevas expectativas en el uso de estos sistemas para casos de hipoacusia neurosensorial no tumoral por malformación congénita de la cóclea (agenesia o hipoplasia severa), aplasia del nervio coclear y osificación total de ambas cócleas ante test de estimulación promontorial negativo.

Agenesia bilateral de cócleas o/y nervios cocleares. A pesar de que ésta es una de las indicaciones emergentes más debatidas, los resultados expuestos y la inexistencia de otras alternativas terapéuticas para la restauración de la audición hizo que se llevaran a cabo las primeras implantaciones de IATC en niños afectos de tales malformaciones ${ }^{43}$. Los resultados comunicados hasta ahora son esperanzadores, y no se ha observado la aparición de complicaciones graves relacionadas con el empleo de estos dispositivos en niños. Indudablemente, el seguimiento a largo plazo de estos pacientes se hace imprescindible antes de generalizar y confirmar la indicación de IATC en la población infantil.

\section{BIBLIOGRAFÍA}

1. National Center for Health Statistics. 1994;188.

2. Abelló P, Martínez Sanjosé J. Prótesis acústicas. En: Gil-Carcedo LM, editor. Tratado de otorrinolaringología y cirugía de cabeza y cuello. Tomo II. 1999. p. 1513-22.

3. Manrique M, Ramos A, López-Villarejo P, García-Ibáñez E. Prótesis implantables en otocirugía. Barcelona: Doyma; 2003.

4. Yanagihara N, Gyo K, Hinohira Y. Partially implantable hearing aid using piezoelectric ceramic ossicular vibrator. Results of the implant operation and assessment of the hearing afforded by the device. Otolaryngol Clin North Am. 1995;28:85-97.

5. Welling DB, Warnes DE. Acoustic stimulation of the semicircular canals. Otolaryngol Clin North Am. 1995;28:207-9.

6. Zenner HP, Leysieffer H, Maassen M, Lehner R, Lenarz T, Baumann J, et al Human studies of a piezoelectric transducer and a microphone for a totally implantable electronic hearing device. Am J Otol. 2000;21:196-204.

7. Kartush JM, Tos M. Electromagnetic ossicular augmentation device. Otolaryngol Clin North Am. 1995;28:155-72.

8. Perkins R. Earlens tympanic contact transducer: A new method of sound transduction to the human ear. Arch Otolaryngol Head Neck Surg. 1996;114: 720-8.

9. Maniglia AJ, Ko WH, Rosenbaum M. A contacless electromagnetic implantable middle ear device for sensorineural hearing loss. ENT J. 1994;73:78-161.

10. Baker RS, Wood MW, Hough JVD. The implantable hearing device for sensorineural hearing impairment: the Hough Ear Intitute experience. Otolaryngol Clin North Am. 1995;28:147-53.

11. Gan RZ, Wood MW, Ball GR, Dietz TG, Dormer KJ. Implantable hearing device performance measured by laser Doppler interferometry. Ear Nose Throat J. 1997;76:297-9.

12. Frederickson JM, Coticchia JM, Khosla S. Ongoing investigations into an implantable electromagnetic hearing aid for moderate to severe sensorineural hearing loss. Otolaryngol Clin North Am. 1995;28:107-20. 
13. Manrique M, Huarte A. Implantes cocleares. Barcelona: Masson; 2002.

14. Torres S, Rodríguez JM, Santana R, González AM. Deficiencia auditiva. Aspectos psicoevolutivos y educativos. Archidona: Aljibe; 1995.

15. Portmann M. Conclusion générale sur la precocité de l'appareillage chez la jeune enfent. Rev Laryng. 1968;89:7-8.

16. Reynier JP. L'appareillage individuel de l'enfent sourd. J F ORL. 1972;21:3.

17. Appaix A, Decroix G, Olivier JC. La prothèse auditive. Paris: Arnette; 1974.

18. Berger KW, Hagberg EN, Rane RL. La adaptacion de prótesis auditivas: fundamentos, método y resultados. Alicante: Estilo; 1986.

19. Magnan J, Manrique M, Dillier N, Snik A, Hausler R. International consensus on middle ear implants. Second Meeting. Consensus on Auditory Implants, 19-21 February 2004, Valencia, Spain. Acta Otolaryngol. 2005;125:1-2.

20. Luetje C, Brackman D, Balkany T, Maw J, Baker S, Kelsall D, et al. Phase III clinical results with the Vibrant Soundbridge implantable middle ear hearing device: A prospective controlled multicenter study. Otolaryngol Head Neck Surg. 2002;126:97-107.

21. Snik AF, Cremers CW. Vibrant semi-implantable hearing device with digital sound processing: effective gain and speech perception. Arch Otolaryngol Head Neck Surg. 2001;127:1433-7.

22. Cochlear Implantation: US Food and Drug Administration PMA P840024/S46 (21/10/96).

23. Manrique M, Cervera-Paz FJ, Huarte A, Molina M. Advantages of cochlear implantation in prelingual deaf children before 2 years of age when compared with later implantation. Laryngoscope. 2004;114:1462-9.

24. Manrique M, Huarte A, Molina M, Cillero G, Ruiz de Erenchun I. Our experience with cochlear implantations in teenagers and prelingually deafened adults. En: Hochmair-Desoyer IJ, Hochmair ES, editores. Advances in cochlear implants. Viena: Mainz; 1994. p. 437-42.

25. Slattery WH, Luxford WM. Cochlear implantation in the congenital malformed cochlea. Laryngoscope. 1995;105:1184-7.

26. Singh RS. Ossified cochlea and its impact on cochlear implants. En: Cochlear implants. Bologna: Monduzzi; 1997. p. 201-3.

27. Lenarz T. Cochlear implants: selection criteria and shifting borders. Acta Otorhinolaryngol Belg. 1998;52:183-99.

28. Seeber BU, Baumann U, Fastl H. Localization ability with bimodal hearing aids and bilateral cochlear implants. J Acoust Soc Am. 2004;116:1698-709.

29. Morera C, Manrique M, Ramos A, García-Ibáñez L, Cavalle L, Huarte A, et al. Advantages of binaural hearing provided through bimodal stimulation via a cochlear implant and a conventional hearing aid: A 6-month comparative study. Acta Otolaryngol. 2005;125:596-606.

30. Real Patronato de Prevención y Atención a Personas con Minusvalía, Comisión de Expertos. Implantes Cocleares. Acta Otorrinolaringol Esp. 2002;53: 305-16.
31. Gstoettner W, Kiefer J, Baumgartner WD, Pok S, Peters S, Adunka O. Hearing preservation in cochlear implantation for electric acoustic stimulation. Acta Otolaryngol. 2004;124:348-52.

32. Schleich P, Nopp P, D'Haese P. Head shadow, squelch, and summation effects in bilateral users of the Med-El Combi 40/40 + cochlear implant. Ear Hear. 2004;25:197-204.

33. Briggs RJS, Brakmann DE, Baser ME, Hitselberger WE. Comprehensive management of bilateral acoustic neuromas. Arch Otolaryngol Head Neck Surg. 1994;120:1307-14.

34. Doyle KJ, Nelson RA. Bilateral acoustic neuromas (Neurofibromatosis 2). En: House W, Lutje CM, Doyle KJ, editores. Acoustic tumor diagnosis and management. San Diego-London: Singular; 1997. p. 308-10.

35. Nevison B, Laszig R, Sollmann WP, et al. Results from a European clinical investigation of the Nucleus multichannel auditory brainstem implant. Ear Hear. 2002;23:170-83.

36. Marangos N, Stecker M, Sollmann WP, Laszig R. Stimulation of the cochlear nucleus with multichannel auditory brainsteam implants and long-term results: Freiburg patients. J Laryngol Otol. 2000;27 Suppl:27-31.

37. Sollman WP, laszig R, Marangos N. Surgical experiences in 58 cases using the Nucleus 22 multichannel auditory brainsteam implant. J Laryngol Otol. 2000;114:23-6.

38. Wu H, Kalamarides M, Bouccara D, Dahan EA, Viala P, Sollmann WP, et al. Auditory brainstem implant (Nucleus 21-channel) in neurofibromatosis type 2 patients previously operated on: preliminary results. Adv Otorhinolaryngol. 2000;57:236-9.

39. Kalamarides M, Grayeli AB, Bouccara D, Dahan EA, Sollmann WP Sterkers $\mathrm{O}$, et al. Hearing restoration with auditory brainstem implants after radiosurgery for neurofibromatosis type 2. J Neurosurg. 2001;95: 1028-33.

40. Green JD, Marion MS, Hinojosa R. Labirinthitis ossificans: histopathologic consideration for cochlear implantation. Otolaryngol Head Neck Surg. 1991;104:320-6.

41. Colletti V, Fiorino FG, Carner M, Miorelli V, Guida M, Colletti L. Auditory brainstem implant as a salvage treatment after unsuccessful cochlear implantation. Otol Neurotol. 2004;25:485-96.

42. Grayeli AB, Bouccara D, Kalamarides M, et al. Auditory brainstem implant in bilateral and completely ossifiedcochleae. Otol Neurotol. 2002;24: 79-82.

43. Colletti V, Carner M, Fiorino FG, Sacchetto L, Orsi A, Cilurzo F, et al. Hearing restoration with auditory brainstem implant in three children with cochlear nerve aplasia. Otol Neurotol. 2002;23:682-93. 